\title{
Structural response of metals to friction-stir welding
}

\author{
S. Yu. Mironov ${ }^{\dagger}$, Y. S. Sato, H. Kokawa \\ ${ }^{\dagger}$ smironov@material.tohoku.ac.jp
}

\author{
Department of Materials Processing, Graduate School of Engineering, Tohoku University, 6-6-02 Aramaki-aza-Aoba, \\ 980-8579, Sendai, Japan
}

The general trends of grain structure development during friction stir welding (FSW) were studied. The microstructure evolution at relatively low welding temperatures was shown to be primarily governed by grain subdivision. At elevated temperatures, however, discontinuous recrystallization may play a key role. Inhomogeneous microstructure distribution was revealed in stir zone.

Keywords: friction stir welding, microstructure, texture.

\section{Introduction}

Friction stir welding (FSW) is a revolutionary joining technique invented in 1991 [1]. The basic concept of FSW is remarkably simple, as shown in fig. 1 .

A non-consumable rotating tool with a specially designed pin and shoulder is inserted into the abutting edges of sheet or plates to be joined and traversed along the line of joint. The tool serves two primary functions: (a) heating of workpeace, and (b) movement of material to produce the joint. The heating is accomplished by friction between the tool and the workpeace and plastic deformation of the workpeace. The localized heating softens the material around the pin and combination of tool rotation and translation leads to movement of material from the front of the pin to the back of the pin. As a result of this process a joint is produced in 'solid state. During FSW process, the material undergoes intense plastic deformation at elevated temperature.

FSW is widely considered as one of the most significant recent achievements in the field of joining. Being a solidstate process, FSW avoids solidification problems associated with conventional fusion welding and thereby yields sound joints, even in materials usually considered to be unweldable. The pronounced advantages of this technology resulted in its widespread usage for joining of various structural materials. In turn, the practical success of the friction stirring techniques necessitated a more fundamental understanding of the underlying physical processes. Thus, microstructural and textural studies are presently one of the key issues in the friction stirring field.

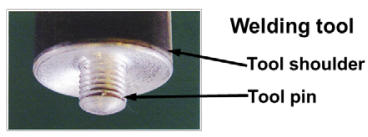

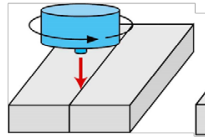

I. Tool rotation

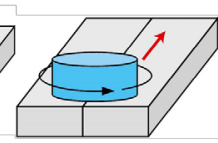

II. Tool plunging

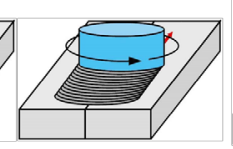

III. Tool travelling

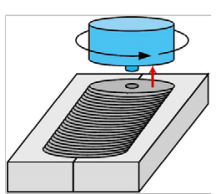

IV. Tool extracting
Fig. 1. Schematic of FSW process.
Our research group is conducting metallurgical studies of microstructures and properties of friction stir welded materials over the last 15 years. Several our achievements in this area are briefly reviewed in this paper.

\section{Crystallographic aspects of material flow}

To evaluate crystallographic aspects of material flow during FSW, texture distribution within stir zone of AZ61 magnesium alloy was studied [2]. To this end, the friction-stir welded material was sectioned perpendicular to welding direction and texture was measured at five different locations, as shown in fig.2. The obtained 0002 pole figures are presented in the bottom part of this figure. It is obvious from the pole figures that the texture distribution was inhomogeneous. Specifically, the basal 0002 planes are nearly parallel to the normal direction (ND) and appear to rotate around this direction when crossing the weld in transversal direction. Assuming that the material flow in the stir zone is mainly associated with the rotating pin, it may be deduced that the basal plane tends to align with the pin column surface. According to the well-accepted conventions, the obtained textural pattern may be described in the terms of so-called B-fiber $\{0002\} / /$ shear plane component of the simple-shear texture.

Two important conclusions were made from these results. First, the material flow in the stir zone appears to be close to the simple-shear deformation. Second, the shear
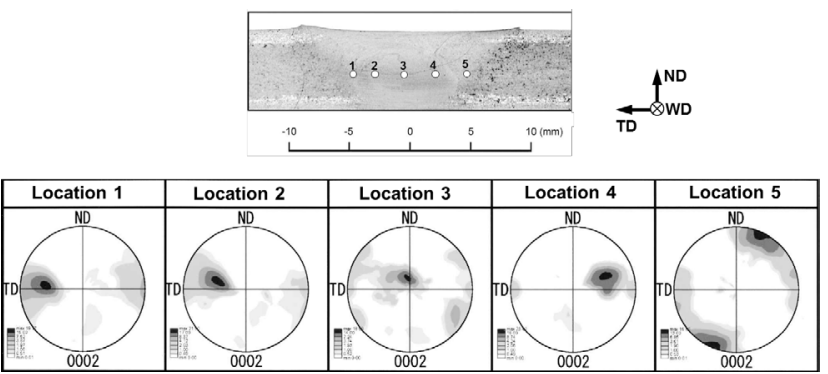

Fig. 2. 0002 pole figures illustrating texture distribution along the weld mid-thickness. WD, TD and ND are welding, transversal and normal directions, respectively (after Park et al. [2]). 
plane/direction are not constant within the stir zone but follow to the column surface of the rotating pin.

It should be also pointed out that the fairly inhomogeneous distribution of the crystallographic texture in the stir zone may have significant impact on mechanical properties of weldments. This effect should be most pronounced in hexagonal metals which are particularly sensitive to the texture.

\section{Specific character of material flow near upper surface}

As shown in fig.1, the welding tool typically consists of pin and massive shoulder; moreover, the shoulder is usually partially plunged into material. It may be expected therefore that the shoulder may exert an influence on the material flow in the upper part of the stir zone. It is usually believed, however, that the shoulder-deformed zone is relatively narrow and this effect thereby may be neglected. In thin sheets, however, this may be not the case and thus the shoulder effect deserves some attention.

To examine the shoulder effect, texture distribution in thickness direction was studied in friction-stir welded AZ31 magnesium alloy [3]. To this end, the friction-stir welded material was longitudinally sectioned parallel to the welding direction along the weld centerline, as shown in fig.3a. For clarity, a tool contour is also superimposed on the figure. From the region behind the tool, a large EBSD map was taken and shown in fig.3b. In the map, individual grains are colored according to their directions relative the welding direction and color code triangle is shown in the top right corner.

It is obvious from the EBSD map that the texture distribution is inhomogeneous in the thickness direction. In the bottom half of the stir zone (red color in the map), the basal 0002 planes are oriented nearly parallel to the tool pin and this is quite expectable result. In the upper part of the weld, however, the basal planes are oriented parallel to the tool shoulder (green color). This also indicates the formation of the simple shear-texture but the shear deformation here is associated with the shoulder but not with the pin.

This study confirmed that the material flow in the upper part of the friction-stir welds is dominated by the shoulder
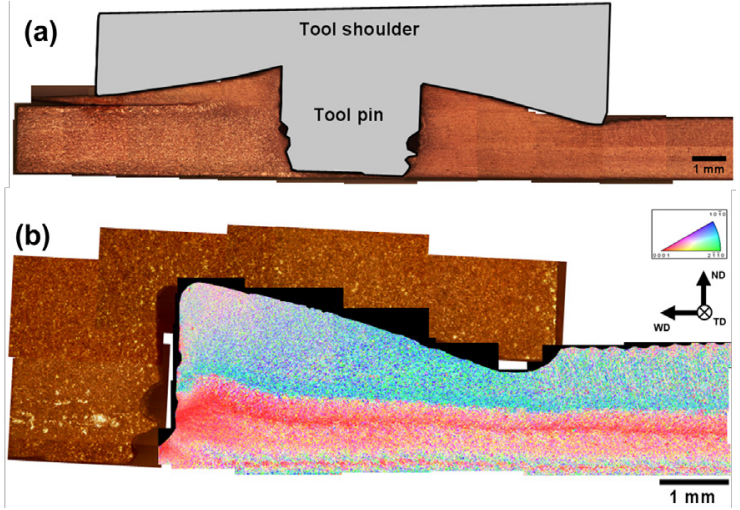

Fig. 3. (a) Low-magnification overview of the longitudinal cross section of the weld (with superimposed contour of the tool) and (b) composite EBSD map taken behind the tool. After Suhuddin et al. [3]. and showed that this effect cannot be ignored in the case of thin sheets.

\section{General trends of grain structure development}

To evaluate fundamentals of grain structure development during FSW, microstructural observations were performed in friction-stirred single crystal austenitic stainless steel [4]. To simulate «cold» and «hot» FSW conditions, the microstructure evolution was studied separately for the pin plunging and shoulder contacting steps. During the pin plunging step, the welding temperature is believed to be relatively low, whereas at the shoulder contacting step it should be relatively high.

Figure $4 \mathrm{a}$ shows a low-magnification overview of the cross section of the weld produced during the pin plunging step. To provide an insight into microstructural evolution, EBSD maps was taken from the location indicated by white rectangle and shown at higher magnification in fig.4b. It is seen from the figure that FSW broke down the initial single crystal orientation into ultra-fine grained structure in vicinity of the pin. To provide a better illustration of this process, selected areas of the large map in fig. $4 \mathrm{~b}$ are given at higher magnification in fig.5. In this figure, low-angle boundaries (LABs) and high-angle boundaries (HABs) are shown as red and black lines, respectively.

The grain structure developed relatively far from the pin keyhole (fig.5a) was dominated by LABs. The traces of the LABs tended to align with the pin surface. The HABs sporadically observed in the microstructure were typically not arranged as an evident grain structure. Instead, they were rather represented by isolated grain-boundary segments (indicated by an arrow in fig.5a). It appears from the structure morphology that the $\mathrm{LAB}$ segments gradually transform into HABs by accumulating misorientation angle. If so, the grain refinement process presumably fits the definition of continuous recrystallization.

Closer to the pin keyhole (fig.5b), HAB fraction significantly increased producing ultra-fine grained structure. Remarkably, the evolved grain structure was still aligned with the pin surface and the mean $\mathrm{HAB}$ spacing in this region was very close to the mean $\mathrm{LAB}$ intercept in fig. $5 \mathrm{a}(\sim 0.4 \mu \mathrm{m})$. It seems therefore that the microstructure evolution was mainly associated with the continuous LAB-to-HAB transformation.

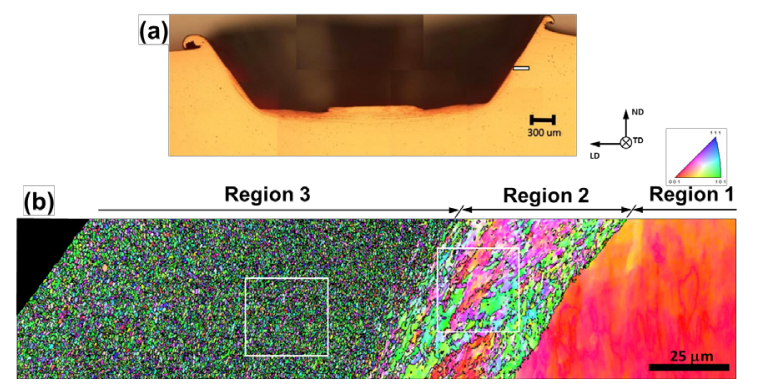

Fig. 4. Pin plunging step: low-magnification overview (a) and EBSD map (b). After Jeon et al. [4]. 


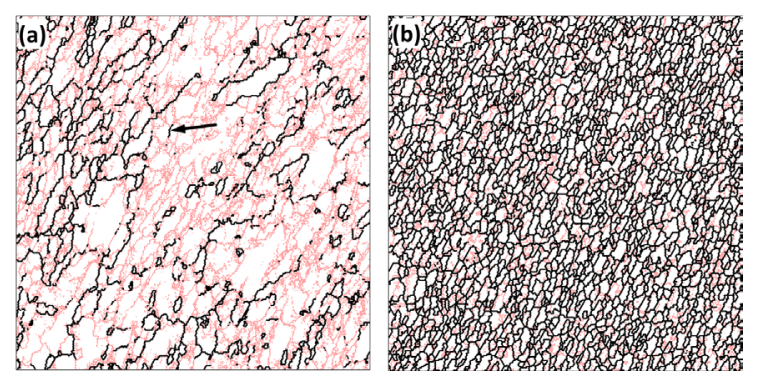

Fig. 5. Selected areas in Fig. 4b shown at higher magnification (after Jeon et al. [4]).
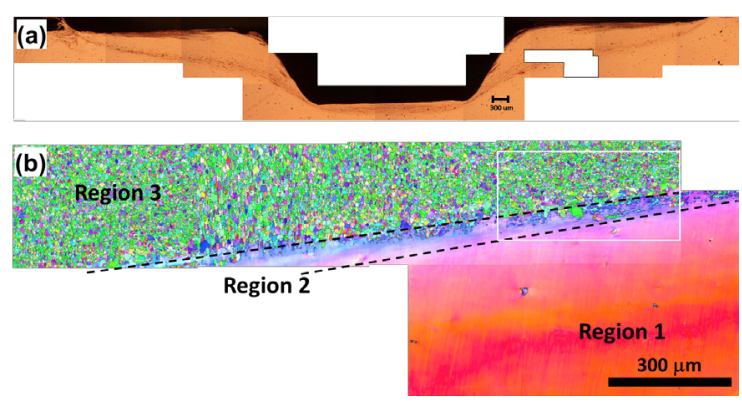

Fig. 6. Shoulder contacting step: (a) low-magnification overview and (b) EBSD map (b). After Jeon et al. [4].

Figure 6a shows a low-magnification optical image of the weld obtained after the shoulder contacting step. At this stage, the shoulder iis believed to exhibit significant contribution to the material flow.

The composite EBSD map acquired from the area marked in fig.6a is given at higher magnification in fig.6b. Relatively far from the tool keyhole, the material was essentially single crystal in nature though orientation spread was obvious in the EBSD map. Near the tool keyhole, however, the single crystal structure was again broken down. To illustrate this process in greater details, a selected area of the large EBSD map in fig.6b is given at higher magnification in fig.7; in this figure, LABs, $\mathrm{HABs}$ and $\Sigma 3$ boundaries are shown as red, black and green lines, respectively.

It is seen that extensive LABs formation became evident approaching to the tool keyhole. Remarkably, the LAB traces tended to align with the shoulder surface. Of particular interest was nucleation of relatively coarse, low-aspect ratio, LAB-free grains. Those grains were characterized by low orientation spread (not shown) and contained annealing twins. It is worth noting that annealing twinning in this material is typically associated with grain boundary migration induced by recrystallization or grain growth. Thus the specific microstructure morphology in this region perhaps indicates the recrystallization driven by nucleation and growth of recrystallization nuclei.

In the vicinity of the tool keyhole, the recrystallized grains significantly increased in volume fraction becoming a predominating microstructural feature; nevertheless, remnants of the deformation matrix were still seen (fig.7). The observed microstructural transformation was accompanied by a notable decrease of LAB fraction. This effect is believed to be mainly attributable to the LAB elimination by the growing recrystallized grains. Generally, the microstructure evidenced an activation of discontinuous recrystallization.

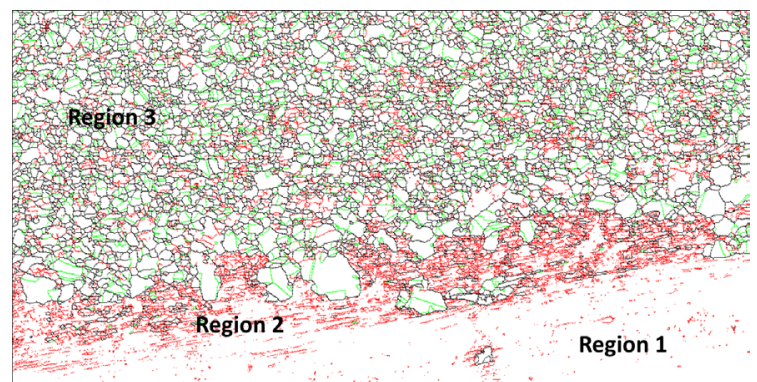

Fig. 7. Shoulder contacting step: grain-boundary map showing microstructural changes approaching tool keyhole (after Jeon et al. [4]).

It may be concluded therefore that at relatively low temperature (simulated here by the pin plunging step), microstructural evolution during FSW may be governed by continuous recrystallization. Considering a very large deformation induced during FSW, this result seems to be reasonable. At relatively high temperatures (simulated by the shoulder contact step), grain boundaries become mobile and thus grain structure development may be driven by discontinuous recrystallization.

\section{Continuous recrystallization}

To examine continuous recrystallization during FSW in greater details, this process was studied in pure iron welded at relatively low temperature [5]. Figure 8 shows a composite EBSD map taken from thermo-mechanically affected zone (TMAZ) of the friction stir weld obtained in this material. In the map, LABs are depicted as white lines, and HABs as black lines.

Near outer edge of the TMAZ, the parent grains tended to be reoriented in a flow pattern around the stir zone due to shear deformation induced by the rotating tool (fig.8a). The extended LABs developed in the grain interiors. They were mainly arranged as regular arrays of nearly parallel bands aligned in a common direction within grains (fig.8b). These bands are referred to as boundary sets.

Closer to the stir zone the original grains became thinner with higher aspect ratios as the strain increased towards the tool (fig.8a). The boundary sets became a dominant feature in the microstructure (fig.8c). Usually, one of the boundary sets was aligned with longitudinal direction of an elongated grain; this set was typically well developed and fairly extended across the grain. Some boundary segments have already accumulated misorientation in excess of $15^{\circ}$ (arrowed in fig.8c).

Near the stir zone, the deformed microstructure became considerably more complicated (fig.8d). Nevertheless, the basic microstructural features seen at lower strains could still be identified. The original grains were drawn out to form the thinly fibrous grains. Simultaneously, the boundaries of primary boundary sets accumulated significant misorientation and broke down the original grain structure forming a lamellar deformation structure. This structure consisted of HABs aligned in a common direction crosslinked by LABs.

The present results demonstrate that the observed microstructural evolution was broadly similar to the 

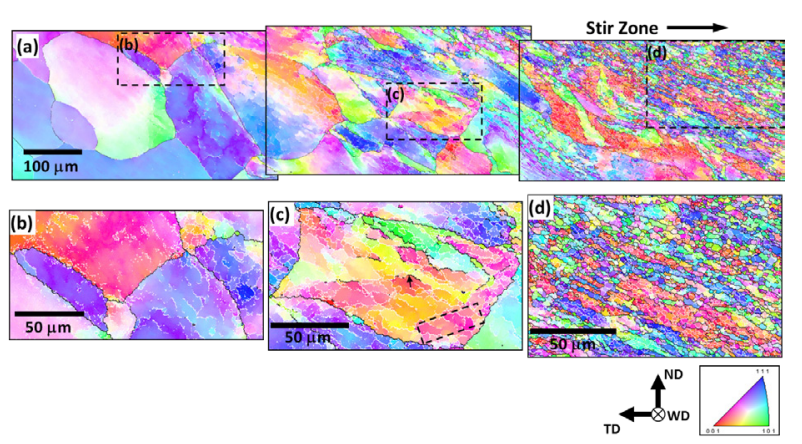

Fig. 8. Composite EBSD map of thermo-mechanically affected zone on retreated side of the friction-stir welded pure iron (a), with selected areas shown at higher magnifications in (b), (c) and (d). After Mironov et al. [5].

universal structural behavior observed in the FCC and BCC metals of medium to high stacking fault energy subjected to cold or warm deformation. The grain structure development process therefore appears to be driven mainly by continuous recrystallization.

\section{Disontinuous recrystallization}

To examine discontinuous recrystallization during FSW, microstructure evolution during high-temperature welding of superaustenitic stainless steel was studied [6]. Figure 9a shows a composite EBSD grain-boundary map obtained from TMAZ and some selected areas are given at higher magnifications in figs.9b-d. Based on morphological differences, the large EBSD map in fig.9a was divided into four regions (Base material, Region 1, Region 2 and stir zone). Misorientation data derived from the microstructural regions are presented in fig. 10.

Near the outer edge of the TMAZ (Region 1), LABs began to develop in grain interiors (fig.9a). Typically, the LABs had very low misorientation (fig.10a). Another important microstructural feature of Region 1 was transformation of twin boundaries. Their misorientations began to deviate from the exact twin/matrix relationship and this process eventually transforms some segments of the twin boundaries into random (not twin) HABs (arrowed in fig.9b). This interesting phenomenon may be explained as being due to the strain-induced crystallographic rotations of the twins and matrix from their initial orientations. To provide a statistical insight into this effect, the deviations of all measured twin boundaries from the ideal $\Sigma 3$ misorientation (within the Brandon interval) were plotted in fig.10b. It is evident from the figure that the deviations significantly increase in Region 1.

In Region 2, the LAB formation became much more pronounced and the subboundaries may be readily seen within a majority of the original grains and twins (fig.9a). The LABs preferentially concentrated near original grain boundaries (fig.9c); their misorientation notably increased and in some cases it exceeded $15^{\circ}$. The process of the straininduced twin destruction continued to develop leading to a significant reduction of the twin boundary fraction.

Additionally, the boundaries of the original grains and twins became essentially wavy. A number of the grain

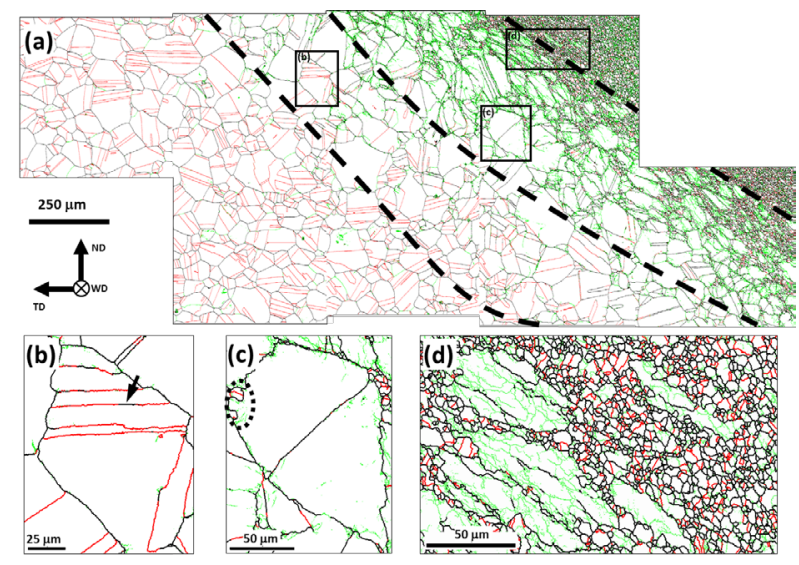

Fig. 9. Composite EBSD map of thermo-mechanically affected zone on retreating side of the friction-stir welded superaustenitic stainless steel (a) with selected areas shown in higher magnifications in (b-d). LABs, HABs and S3 boundaries are depicted as green, black and red lines, respectively. After Mironov et al. [6].
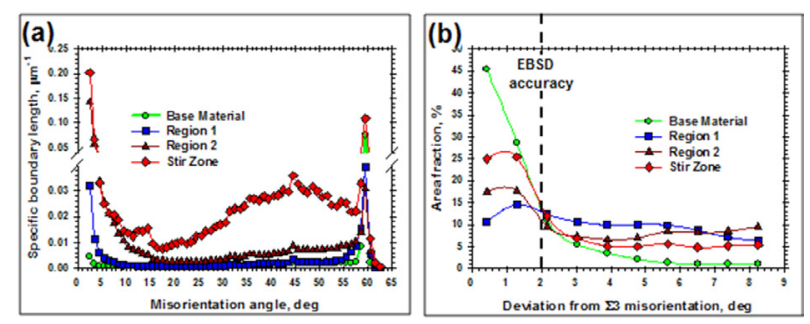

Fig. 10. Misorientation data derived from different regions in Fig. 9a: specific grain-boundary area statistics (a) and deviations of the measured twin boundary misorientations from ideal $\Sigma 3$ (b). After Mironov et al. [6].

boundaries exhibited local bulges, some of which appear to convert into fine equiaxed grains (an example is shown as selected area in fig.9c). This process substantially increased the $\mathrm{HAB}$ area in the misorientation range of $\sim 15-55^{\circ}$ (fig.10a). Interestingly, the migrating grain boundary segments frequently left behind twins (fig.9c, encircled). Misorientation of these newly developed twins was frequently within $2^{\circ}$ tolerance of the ideal $\Sigma 3$ and this increases the area fraction of the nearly perfect twins in the microstructure (fig.10b). These observations indirectly indicate annealing twinning.

Near the stir zone extremity, the microstructure became considerably more complicated. The original grains tended to be geometrically reoriented and sheared (fig.9d). LAB area further enlarged, especially in the range of $10-15^{\circ}$ (fig.10a). The LAB morphology principally changed (fig.9d). Their distribution became much more homogeneous in a grain scale and there was no tendency for the LAB clustering near original grain boundaries anymore. The LABs became more extended and straight, and their extended segments accumulated misorientation over $15^{\circ}$, thus transforming into HABs. Frequently the LABs were arranged as more or less regular arrays of roughly parallel bands aligned in a longitudinal direction of grains. These observations may be interpreted in the terms of the continuous recrystallization.

On the other hand, the process of the bulging of the original grain-boundaries and associated formation of fine equiaxed grains led to the creating of a necklace structure 
decorating elongated parent grains (fig.9d). The newly developed fine grains were characterized by a very low orientation spread (not shown), were completely surrounded by $\mathrm{HABs}$ and typically contain almost no substructure with the exception of annealing twins (fig.9c). Therefore, all these microstructural changes appear to fit the definition of the discontinuous recrystallization controlled by the local migration of the original grain boundaries and accompanied by the annealing twinning.

Thus the grain structure evolution at the stir zone extremity appeared to be a competition between the continuous and discontinuous recrystallization. It is interesting to know therefore what process was dominant.

The evolution of the specific grain-boundary area statistics (fig.10a) may provide an insight into this issue. The misorientation distribution clearly shows a drastic increase of the $\mathrm{HAB}$ area in the stir zone. It is also evident that this process did not develop via progressive movement of the low-angle peak towards high-angle misorientations, but rather by the build-up of a wide peak centred near $45^{\circ}$. This perhaps means that it was unlikely that the continuous recrystallization (assuming a gradual increase of the dislocation boundary misorientation) played a major role in the observed $\mathrm{HAB}$ area enlargement. It may be hypothesized that this effect was rather associated with the discontinuous recrystallization involving local migration of the original grain boundaries and therefore this process may be a key mechanism governing the microstructure formation.

\section{Microstructure distribution in stir zone}

As shown in Sections 2 and 3, texture distribution may be very imhomogeneous in stir zone. To examine possible variations of grain structure, EBSD maps were taken from various locations within stir zone of friction-stir processed single crystal austenitic steel [7], as shown in fig.11. In the maps, LABs, HABs and $\Sigma 3$ boundaries are depicted as red, black and green lines, respectively.

In the upper part of the stir zone (locations 1 to 3 ), a narrow fine-grained layer, which was directly adjacent to the top surface, was found. The material in this area was characterized by a relatively large LAB fraction and low content of $\Sigma 3$ twin boundaries. The formation of this microstructural region is thought to be related to the specific character of material flow during FSW. As demonstrated in Ref. [3], the rotating tool shoulder may entrap the surface layer of the base material and deposit it far behind passed pin. The deposited material should experience perhaps the largest strain at the highest strain rate. This may give rise to a surface layer with a very heavily deformed grain structure, as found in the present study. An alternative explanation for the development of this microstructure may be a relatively high cooling speed near the upper surface (enhanced by air convection) which suppressed recrystallization.

At the midthickness of the stir zone (locations 4 to 6), the evolved grain structure was characterized by the largest grains size, a relatively low proportion of LABs and the highest fraction of $\Sigma 3$ annealing twin boundaries. In other words, the microstructure here was most recrystallized in appearance. This effect may be related to the longest exposure

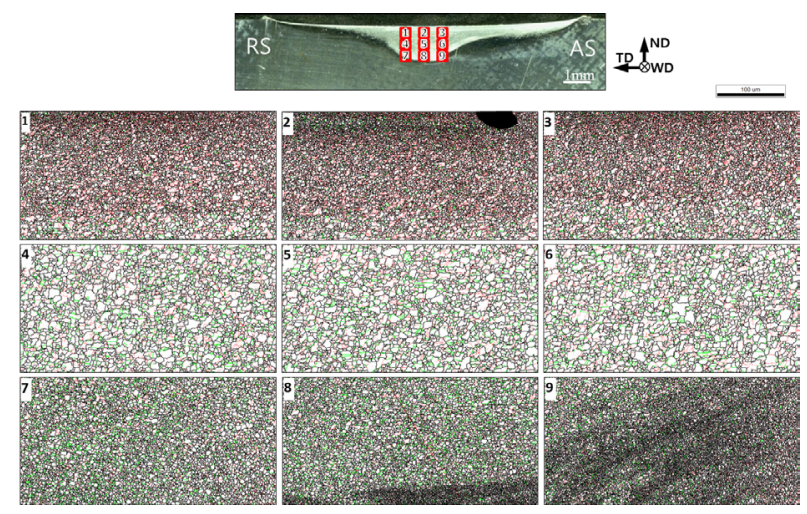

Fig. 11. Selected portions of grain-boundary maps showing microstructures in 9 different locations within the stir zone. In the maps, LABs, HABs and $\Sigma 3$ twin boundaries are depicted as red, black and green lines, respectively. After Jeon et al. [7].

at high temperatures. It is worth noting that thermal conductivity of austenitic stainless steel is comparatively low and thus the FSW cooling cycle may be relatively long. As a result, recrystallization process in this region may be most completed.

In the bottom part of the stir zone (locations 7 to 9), the grain structure again became somewhat finer and the fraction of the annealing twins slightly decreased. This effect is thought to be attributable to a rapid heat sink into the backing plate. Moreover, the development of very fine grains was evident (locations 8 and 9). The microstructure which evolved in this area was virtually bimodal and could be described in terms of a mixture of relatively coarse and fine grains. This phenomenon is thought to be related to local precipitation of the sigma phase in this area [8].

It could be concluded from this work that grain structure in the stir zone may significantly vary in thickness direction due to both specific character of material flow as well as variation of cooling speed

\section{Phase transformation in Ti-6Al-4V alloy}

In some materials, phase transformations may occur during FSW thermal cycle. They may principally change microstructure and thus fundamentally affect weld properties. To improve the basic physical understanding of this phenomenon, microstructural changes in friction-stir welded Ti-6Al-4V alloy was studied [9].

Figure 12a shows a typical EBSD phase map taken from the central part of stir zone. It is seen that the dominant $a$ phase exhibits a basketweave microstructure. The structure morphology evidences that FSW peak temperature exceeded $\beta$-transus (the temperature at which low-temperature a phase transforms into high-temperature $\beta$ phase) and the final microstructure in the stir zone was formed via $\beta$-to- $\alpha$ phase transformation during FSW cooling cycle.

To examine the orientation relationship between the $\alpha$ and $\beta$ phases, local crystallographic orientations of both phases were compared with each other. A typical example of the analyzed area is shown in fig.12b; $\alpha$ grains whose orientations were compared with those of the $\beta$ particle are numbered $1-7$. The obtained orientation data are summarized in 

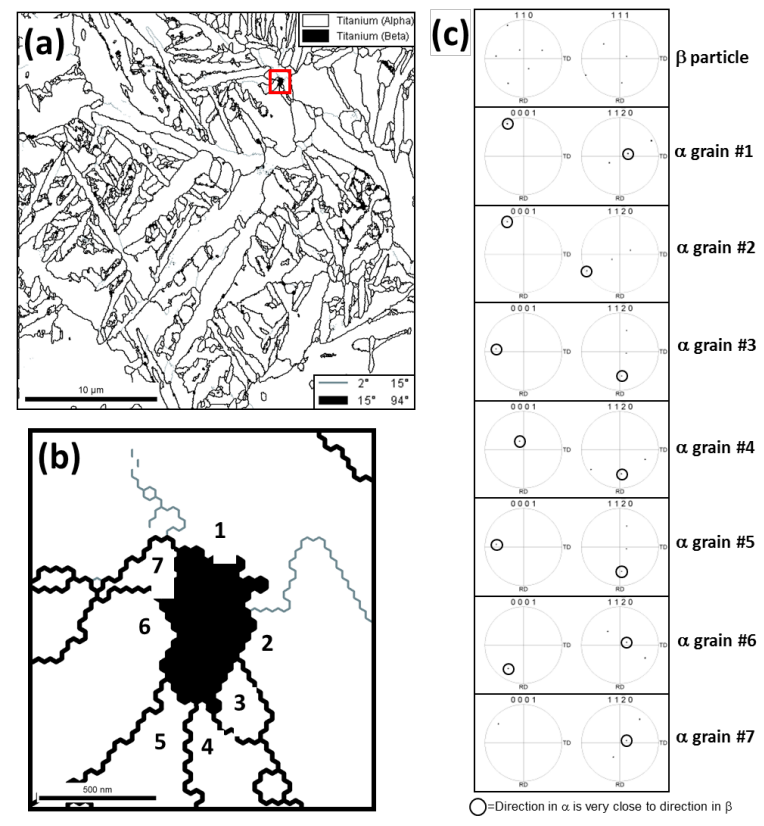

Fig. 12. (a) EBSD phase map with selected area shown at higher magnification in (b); (c) orientation of $\beta$ particle and $\alpha$ grains numbered $1-7$ in (b). After Mironov et al. [9].

fig. $12 \mathrm{c}$ as $\{110\}$ and $\{111\}$ pole figures for the $\beta$ phase and as $\{0002\}$ and $\{11 \overline{2} 0\}$ pole figures for the $\alpha$ phase. For simplicity, crystallographic directions in the $\alpha$ phase that are close to those in the $\beta$ phase are encircled. It is seen that the $<0001>$ and $<11 \overline{2} 0>$ directions of $\alpha$ grains typically are nearly parallel to the $\langle 110\rangle$ and $\langle 111\rangle$ directions of the $\beta$ phase, respectively. This indicates that these $\alpha$ grains and $\beta$ phase nearly satisfy the Burgers orientation relationship (OR) defined by $\{0001\} \alpha / /\{110\} \beta$ and $<11 \overline{2} 0>\alpha / /<111>\beta$.

To provide a statistically significant insight into the phase transformation, misorientation distribution in the a phase was measured and shown in fig.13. The misorientation-angle distributions had a very complex shape exhibiting three sharp peaks: one near $10^{\circ}$, one near $90^{\circ}$ and a split peak in the vicinity of $60^{\circ}$ (fig.13a). Misorientation axes (fig.13b) formed one cluster around the $<0001>$ pole and one broad cluster near the $<2 \overline{1} 10>$ pole. The cluster near the $<0001>$ pole is composed primarily of boundaries with misorientation angles ranging from $5^{\circ}$ to $15^{\circ}$, whereas the clusters near the $<2110>$ pole are produced mainly by the boundaries in the angular ranges of $55^{\circ}-65^{\circ}$ and $80^{\circ}-90^{\circ}$ (fig. $13 \mathrm{~b}$ ).

The misorientation distributions are very close to those predicted for the Burgers OR [10]. Therefore, the misorientation distribution for the $\alpha$-phase additionally confirms that crystallography of the $\alpha$ and $\beta$ phases in the stir zone were related via the Burgers OR.
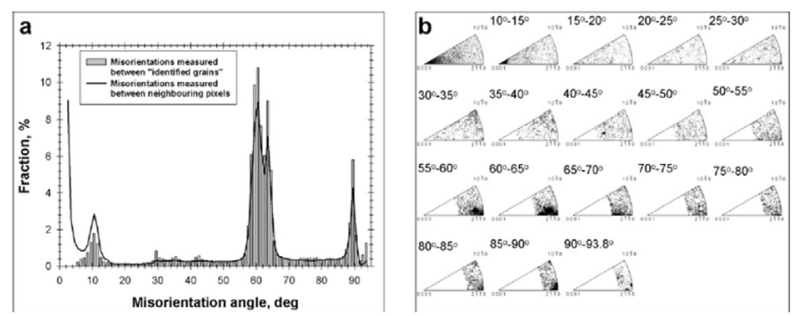

Fig. 13. Misorientation data taken from the $\alpha$ phase: (a) misorientation angle distribution and (b) misorientation axis distribution (after Mironov et al. [9]).

\section{Conclusions}

Material flow during FSW is close to simple shear deformation. The material flow is governed by a combined effect of tool pin and tool shoulder. Rotation of the tool creates a very characteristic heterogeneity in texture distribution in the stir zone. Specific character of material flow in the upper part of the stir zone may give rise to a very fine-grained layer in near-surface region.

Development of grain structure during FSW is complex process driven by a combination of continuous and discontinuous recrystallizations. Contribution of each mechanism may depend on welding temperature.

The results shown in this work were published in $1^{\text {st }}$ International Joint Symposium on Joining and Welding 6-8 November 2013, Woodhead Publishing, 2013

\section{References}

1. W.N. Thomas. Friction stir butt welding. Int. Patent No. PCT/GB92/02203-1991.

2. S.H.C. Park, Y.S. Sato, and H. Kokawa. Metall. Mater. Trans. A. 34, 988 (2003).

3. U.F. H. R. Suhuddin, S. Mironov, Y.S. Sato, H. Kokawa, C.-W.Lee. Acta Mater. 57, 5406 (2009).

4. J. Jeon, S. Mironov, Y.S. Sato, H. Kokawa, S. H.C. Park, S. Hirano. Metall Mater. 59, 7439 (2011).

5. S. Mironov, Y. S. Sato, H. Kokawa. Metall Mater. 56, 2602 (2008).

6. S. Mironov, Y.S. Sato, H. Kokawa. Acta Mater. 59, 5472 (2011).

7. J. Jeon, S. Mironov, Y.S. Sato, H. Kokawa, S.H.C. Park and S. Hirano. Metall. Mater. Trans. A. 44, 3157 (2013).

8. S.H.C . Park, Y. S. Sato, H. Kokawa, K. Okamoto, S. Hirano, M. Inagaki. Scripta Mater. 49, 1175 (2003).

9. S. Mironov, Y. Zhang, Y.S. Sato and H. Kokawa. Scripta Mater. 59, 511 (2008).

10. N. Gey, M. Humbert. Acta Mater. 50, 277 (2002). 